\title{
Validez de la Escala de Confianza en la Tutoría Afectiva y utilidad de la tutoría académica
}

\section{Validity of the Confidence Scale in Affective Tutoring and the usefulness of Academic Tutoring}

DOI: https://doi.org/10.32870/dse.v0i24.1080

\author{
Laura Karina Castro Saucedo* \\ Laura Fabiola Núñez Udave** \\ Cirilo Humberto García Cadena*** \\ Esmeralda Jaqueline Tapia García**** \\ César Arnulfo de León Alvarado*****
}

\begin{abstract}
Resumen
El objetivo de esta investigación fue validar una escala de tutorías identificando dos factores: 1. confianza en la tutoría afectiva y 2 . utilidad en la tutoría académica. El estudio siguió una metodología cuantitativa con el diseño de un instrumento tipo Likert validado con una muestra de 343 estudiantes universitarias en Coahuila y Aguascalientes, México. Las limitaciones del estudio estuvieron relacionadas con la representatividad, enmarcada sólo en la población participante. La originalidad del manuscrito radica en su aportación metodológica y la propuesta de un instrumento de medida validado para determinar la implicación de la tutoría universitaria. Los resultados arrojan una adecuada validez y confiabilidad en cada una de sus dimensiones: Confianza en la Tutoría Afectiva (varianza explicada $=44.67 \%$; Alpha de Cronbach $=.88$ ), Utilidad en la Tutoría Académica (varianza explicada $=59.14 \%$; Alpha de Cronbach $=.85$ ) obteniendo un modelo con índices adecuados (CFI =.932; TLI =.906; IFI = .933, RMSEA =.08).
\end{abstract}

Palabras clave: tutoría - afectividad - académica - confianza - utilidad.

\section{Abstract}

The objective of this research was to validate a tutoring scale by identifying two factors: 1 . confidence in affective tutoring and 2. usefulness of academic tutoring. The study followed a quantitative methodology with the design of a validated Likert-type instrument and a sample of 343 university students in the states

* Doctora en Filosofía con orientación en Trabajo Social y políticas comparadas de bienestar social. Líneas de investigación: Psicología educativa, psicología social, teoría social, y perspectiva de género. Profesora de tiempo completo en la Facultad de Trabajo Social, Universidad Autónoma de Coahuila. México. karinacastro@uadec.edu.mx

** Doctora en Ciencias de la Educación. Líneas de investigación: Educación social, Ciencia de la Educación, grupos vulnerables y en riesgo social. Profesora de tiempo completo en la Facultad de Trabajo Social, Universidad Autónoma de Coahuila. México. lauranunezudave@uadec.edu.mx

*** Doctor en Política Social. Líneas de investigación: Cognición-educación, Psicología conductista, Construccionismo social. Profesor-investigador de tiempo completo en la Facultad de Psicología de la Universidad Autónoma de Nuevo León. México. ciriloenator@gmail.com

**** Doctora en Ciencias de la Educación. Líneas de investigación: Educación social, Trabajo social educativo, grupos vulnerables y en riesgo social. Profesora de tiempo completo en la Facultad de Trabajo Social, Universidad Autónoma de Coahuila. México. estapiag@uadec.edu.mx

***** Doctor en Educación en el Desarrollo de Habilidades Emocionales. Líneas de investigación: Filosofía de la educación, Competencias socioemocionales. Profesor-investigador de tiempo completo en la Facultad de Trabajo Social, Universidad Autónoma de Coahuila. México. cesar.leon@uadec.edu.mx 
of Coahuila and Aguascalientes, Mexico. The limitations of the study were linked to its representativeness, limited to the participating population. Its originality lies in its methodological contribution and the proposal of a validated measurement instrument to determine the implications of university tutoring. Our results show adequate validity and reliability in each of its dimensions: Confidence in Affective Tutoring (explained variance $=44.67 \%$; Cronbach's Alpha $=.88$ ), Usefulness in Academic Tutoring (explained variance $=59.14 \%$; Cronbach's Alpha $=.85)$, yielding a model with adequate indices (CFI $=.932 ; \mathrm{TLI}=.906$; IFI $=.933$, RMSEA $=.08$ ).

Keywords: tutoring - affectivity - academic - confidence - utility.

\section{Introducción}

La tutoría es entendida como una relación que se da cara a cara entre el docente que funge como tutor y el alumno o tutorado, en la que se procura formar al tutorado para que enfrente las problemáticas que se le presentan en su vida escolar y llegue a resoluciones favorables que le aseguren el éxito escolar y profesional (Canales, 2010). Sin embargo, esta concepción se ve limitada, considerando que este proceso se encuentra con diversas situaciones emergentes derivadas de los contextos posmodernos en los que interactúa el tutorado y el tutor, trayendo consigo la implicación de atender no sólo las cuestiones académicas sino también las personales, sociales y de otra índole. En este escenario, González y Avelino (2016) proponen cambiar el término de tutoría de forma individual al de tutorías, de manera plural, haciendo referencia a que el ejercicio de la tutoría no sólo atiende cuestiones académicas, ni se da en una sola tutoría, sino que es un proceso continuo, constante y variado, que incluso no sólo se da cara a cara, sino que en la actualidad puede presentarse a través de diversos medios de comunicación dependiendo de las necesidades y los contextos de los tutorados y los tutores.

Con respecto a la evaluación de la efectividad de la tutoría, Guerra, Lima y Lima (2017) realizaron una investigación con universitarios de enfermería en Sevilla, España, cuyos resultados muestran la efectividad del programa de tutoría al reforzarla como una estrategia fundamental para lograr el alcance de metas. En los resultados de la investigación realizada por Zaldívar, Nava y Lizárraga (2018) en educación media superior con estudiantes de un bachillerato se resalta que, de acuerdo con la percepción de los participantes, los tutorados perciben disposición, confianza y respeto en el tutor, pero consideran una debilidad en los tutores el conocimiento en la materia y el dominio pedagógico.

En sus investigaciones, Andreucci y Curiche (2017) presentan una serie de desafíos que enfrenta el tutor para garantizar la tutoría efectiva en sus tutorados; estos desafíos tienen que ver con la cobertura del programa, las estrategias utilizadas para la captación de la motivación y la persistencia del estudiante, así como el acompañamiento y la focalización en las necesidades de cada uno de sus tutorados. En el caso de la Universidad de Burgos, España, la tutoría académica 
se trabaja con respecto a tres facetas: 1) académica o de apoyo a los procesos de enseñanzaaprendizaje del alumno, 2) curricular o de orientación a la hora de elegir su itinerario académico y profesional, y 3) personal o de asesoría respecto a su desarrollo integral. Beascoa y Moreno (2016) elaboraron una escala con 16 ítems sobre actitudes de los estudiantes hacia las tutorías académicas, de la cual los resultados mostraron la existencia de las tres dimensiones previstas en la estructura teórica; así se puede entender que la actitud de los estudiantes viene determinada por tres aspectos: 1) seguridad o confianza, 2) motivación o interés personal, y 3) utilidad para la asignatura.

De igual forma, Caldera, Carranza, Jiménez y Pérez (2015) realizan un estudio cuya finalidad es la validación de la Escala de Actitudes Hacia la Tutoría (EAHC); dentro de los resultados concluyeron con una escala validada conformada por 16 ítems con tres factores correlacionados: 1) Creencias sobre la tutoría, 2) Afectos sobre la tutoría, y 3) Disposiciones ante la tutoría. En un estudio presentado por Gómez (2012), el cual midió la percepción de los estudiantes sobre el Programa de Tutoría Académica en la Universidad Autónoma del Estado de México, dio cuenta de que existen diferencias importantes entre carreras, ya que las licenciaturas de Ciencias Políticas y Administración Pública participan con mayor entusiasmo, a diferencia de la licenciatura en Comunicación. En otro estudio realizado por Rubio y Martínez (2012), con alumnos de la Universidad Autónoma de San Luis Potosí, en el cual los mismos estudiantes construyen sus concepciones y expectativas, y analizan las implicaciones de la acción tutorial de los programas al respecto implementados en su institución, los resultados muestran que las problemáticas operacionales que más inciden en la implementación de estos programas tienen que ver con la organización y la disposición del tutor para impartir las actividades tutoriales.

La aplicación responsable de la tutoría permitirá conocer las características individuales, los intereses personales, las expectativas profesionales y laborales, y los proyectos de vida de los jóvenes, y con ello establecer relaciones interpersonales entre el profesor-tutor y el estudiante, que redunden no sólo en beneficio de ambos, sino también del ambiente en el que se desarrollan (Ayala, Naranjo, Castro, 2014). Desde estos abordajes es posible comprender para este estudio como tutoría afectiva al acercamiento personalizado de consejería y acompañamiento desde la confianza, el respeto y el afecto recíproco entre el tutor y el tutorado. De esta manera, la revisión de la tutoría afectiva es la iniciativa de plasmar una tutoría que no sólo se limite a los quehaceres administrativos y académicos, sino que trascienda en situaciones de la vida personal de los tutorados. En un estudio realizado por Sánchez, Amador y Castillo (2011), encontraron que el programa de tutorías obtiene aciertos en la dimensión consejería académica y formación investigadora.

En otro estudio realizado por Ayala, Naranjo y Castro (2014), donde se evaluó el programa institucional de tutorías, se obtuvo como resultado que la mayoría de las veces los tutores no asumen la acción tutorial con pasión, responsabilidad y compromiso, volviéndose esta una ac- 
tividad más que les aporta puntaje para la beca al desempeño académico. Ahumada y Obregón (2015) señalan que la autoestima es fundamental en el éxito del desempeño académico, por tanto, todo aquel docente que se concibe como tutor no deberá perder de vista en su trabajo favorecerla desde el proceso formativo que refieren los pilares de la educación.

Por otra parte, Romero, Chávez y Sandoval (2014) mencionan que se ha propuesto aumentar los servicios de apoyo a los docentes y estudiantes universitarios, entre los cuales se destaca una educación más personalizada a través de programas institucionales de tutoría. Asimismo, en el contexto del proceso de enseñanza-aprendizaje y con el propósito de apoyar al estudiante en su formación, aparece la figura del profesor-tutor, el cual tiene como propósito apoyar al estudiante en su trayectoria académica para lograr egresados competentes en el programa educativo en el que se encuentren estudiando. Para Hernández, Martínez y Carranza (2013), la tutoría académica surge como una alternativa para tratar de prevenir problemas de reprobación y deserción, al facilitar la adaptación del estudiante al ambiente escolar y mejorar sus habilidades de estudio y trabajo, aumentando la probabilidad del éxito en sus estudios.

\section{Validez de escalas de tutorías}

El estado actual que guardan los instrumentos y escalas sobre tutoría responden a diversos modelos, enfoques y experiencias de implementación de los programas, prácticas y procesos de tutoría en el ámbito nacional e internacional y con características que también dependen del nivel educativo donde se implementan. Desde una revisión sistemática de la tutoría de educación superior a nivel nacional, la Universidad de Guadalajara diseñó una escala de medición sobre las actitudes de los estudiantes universitarios ante la tutoría. Para el cumplimiento del objetivo, se diseñó y validó la Escala de Actitudes Hacia la Tutoría (EAHC), la cual mostró propiedades psicométricas satisfactorias; la muestra estuvo integrada por 539 estudiantes. El instrumento se sometió a un proceso de validación de contenido, se consultó a cinco profesores universitarios que fungieron como expertos en la revisión y recomendaciones al mismo, posteriormente se efectuaron cambios en la redacción de la escala. Este procedimiento permite profundizar en el enfoque de la tutoría, al igual que las siguientes dos etapas que construyeron los autores al evaluar la comprensión de los reactivos del instrumento mediante una prueba piloto con 10 estudiantes, y la aplicación del instrumento a 539 estudiantes de las cuatro carreras antes comentadas.

Con el objetivo de identificar el poder discriminativo de los reactivos, se realizaron una serie de pruebas $t$ cuyos valores se obtuvieron al comparar las medias obtenidas por cada uno de los reactivos de los sujetos de los grupos "bajo" (27\% de sujetos con puntuaciones más bajas en la escala) y "alto" ( $27 \%$ de sujetos con puntuaciones más altas en la escala). Finalmente, el instrumento fue sometido al proceso de validación de constructo, para lo cual se realizaron tanto el análisis factorial exploratorio (con la primera mitad de la muestra) como el confirmatorio 
(segunda mitad). Posterior a la validación, se calculó la confiabilidad de la escala y se identificaron frecuencias y medias de la población estudiada. Este procedimiento plasma un enfoque nacional de la tutoría y medición.

En la Universidad Autónoma de México se realizó la construcción de un instrumento de tutorías con la revisión de funciones tanto formativas como socializadoras, así como aspectos interpersonales que promueven la interacción entre posgraduados y tutores. La validez de las mismas se obtuvo mediante análisis factoriales, cada escala explica más de $50 \%$ de la varianza (De la Cruz, Abreu, 2017).

Desde una revisión sistemática de la tutoría en educación superior a nivel internacional, López y Pantoja (2016) presentan el proceso de elaboración y validación de una escala que permite conocer el grado de satisfacción que tienen los maestros de esta etapa educativa en Andalucía al ejercer la tutoría. Para ello se diseñó una escala de 44 ítems que fue aplicada a 1,755 tutores andaluces. Se realizó un análisis de fiabilidad mediante el Alpha de Cronbach (.916), así como validez de contenido y de constructo, aportando esta última una agrupación de ítems en torno a cinco dimensiones.

El trabajo realizado por Pérez, Martínez y Martínez (2015) presenta el diseño y validación de una escala de satisfacción con la tutoría y el grado de satisfacción en una muestra de estudiantes $(n=976)$. El análisis factorial es satisfactorio, la escala queda compuesta por 22 reactivos y dos subescalas que explican $57.87 \%$ de la varianza. La fiabilidad del instrumento $(a=.973)$ y de las subescalas ( $a=.967$ y $a=.944$ ) es alta. Los resultados muestran una mayor satisfacción con el tutor que con la organización y contenidos de la tutoría. Por último, un estudio realizado en 2015 aporta evidencias de validación de una escala de valoración destinada a evaluar las estrategias de gestión e intervención docente, entre ellas la tutoría, también llamadas metodologías docentes, empleadas por el profesorado de primaria. La validación de dicha escala se realizó con un total de nueve centros de la Comunidad Valenciana, España, en el nivel de 60 grado de primaria, concretamente 324 estudiantes. Los resultados comprueban el alto nivel de consistencia interna de la escala de valoración y, por otro lado, presentan un instrumento destinado a la autoevaluación y/o heteroevaluación que posibilite al docente la información necesaria para modificar y mejorar su gestión e intervención en el aula, para adaptarse mejor a las competencias y necesidades de sus estudiantes en un contexto de formación permanente (Chiva, Ramos, 2015).

En la Universidad Complutense de Madrid se aplicó un instrumento elaborado para valorar el funcionamiento de las tutorías por parte de los estudiantes. Estuvo formado por cuatro dimensiones: Funciones del tutor con el alumnado, Funciones del tutor con las familias, Desarrollo de la tutoría y Evaluación. El cuestionario está configurado por 37 preguntas en escala tipo Lickert. Para obtener evidencias de validez de contenido del instrumento en relación con la fundamentación teórica, se utilizó el procedimiento de juicio de expertos (Martínez-Arias, 2005). 
Una vez recogidas las respuestas del juicio de expertos, se seleccionaron los reactivos con una puntuación media igual o menor que 4, y aquellos que tuvieran una desviación típica mayor de 1.5 (Cortada, 1999). Algunos reactivos fueron eliminados, y los reactivos finales presentan medias que oscilan entre 4.13 y 4.88 , así como desviaciones típicas entre 0.354 y 1.458 . Este estudio destaca los modelos de tutoría basados en sus funciones, más que en su afectividad o individualidad. Se estudió la fiabilidad del instrumento mediante el cálculo del Alpha de Cronbach, así como el análisis de la capacidad de discriminación de los ítems a través del índice de homogeneidad. Este indicador refleja el grado que un reactivo mide lo mismo que la prueba global. Los valores inferiores a 0.2 determinan que se suprima el ítem (Hair et al., 2014). De igual modo, para la validación del constructo se determinó el ajuste de bondad del modelo inicial propuesto a nivel teórico, a través del IBM SPSS AMOS versión 21. Se utilizó el Análisis Factorial Confirmatorio (AFC), para cuya aplicación e interpretación nos basaremos en las indicaciones de Byrne (2010), Kline (2010) y Hair et al. (2014): CMIN/DF entre 2 y 5, CFI e IFI > 0.9, PRATIO, PNFI y PCFI > 0.7, RMSEA < 0.06, y HOELTER > 200). Por otro lado, el estudio de la fiabilidad se ha obtenido a través de la consistencia interna del instrumento, calculando el Coeficiente de Alfa (a) de Cronbach, el cual fue de 0.927. Se estudió la fiabilidad, de acuerdo a los resultados del AFC, obteniendo un valor final muy bueno y valores aceptables por dimensiones (a global: 0.929, a dimensión Funciones con el alumnado: 0.868 , a dimensión Funciones con las familias: 0.649 , a dimensión Desarrollo: 0.797, a dimensión Evaluación: 0.876). Este estudio refleja una herramienta de bastante utilidad para los centros educativos y sus profesionales, con la posibilidad de atender las demandas y necesidades de los estudiantes (León, Fernández, 2019).

En la Universidad de Burgos, se realizó un trabajo cuyo propósito fue elaborar una escala de actitudes de los estudiantes hacia las tutorías académicas, que permita conocer, monitorear o mejorar la función tutorial. El estudio se ha ceñido a estudiantes de carreras de ingeniería y como resultado se ha obtenido una escala de 16 ítems. Distintas pruebas y medidas han puesto de manifiesto su consistencia interna y fiabilidad. Tras un análisis factorial con rotación de factores, se han identificado tres dimensiones: aspectos de seguridad o confianza, de motivación o interés personal y de utilidad para la asignatura. Este instrumento denota un modelo de tutoría basado en aspectos de seguridad y confianza, motivación e interés personal en la tutoría. El cuestionario se aplicó al mayor número posible de alumnos de la Escuela Politécnica Superior de la Universidad de Burgos de distintas titulaciones adaptadas al EEES. Se eligió el cambio de semestre como momento idóneo para su pase, por considerar que en ese punto del curso los alumnos tienen reciente la visión global del desarrollo de una asignatura. Participaron voluntariamente 239 estudiantes de todos los cursos y titulaciones técnicas impartidas en dicha universidad. Finalmente, con los datos obtenidos del cuestionario se realizaron pruebas de validez de constructo y medidas de fiabilidad, como el análisis de la matriz de correlaciones, extracción y rotación de factores, interpretación de factores, modelo factorial y medidas de fiabilidad y 
homogeneidad. De los resultados se puede concluir que la actitud de los estudiantes viene determinada por aspectos de seguridad o confianza, de motivación o interés personal y de utilidad para la asignatura. Así, confirman la buena concepción que respecto de la tutoría tiene el alumnado como herramienta de aprendizaje y mejora de su rendimiento académico, como comentan Tejedor y García-Valcárcel (2007); la inseguridad y desinterés hallados explican la escasa asistencia a las tutorías, detectada por Lobato et al. (2005) o Rumbo y Gómez (2009); en definitiva, vienen a confirmar la ausencia de una cultura de acción tutorial que mencionan estos y otros muchos autores como Michavila y García (2003), Vieira y Vidal (2006) o el mismo informe Bricall (2000).

A nivel nacional, los modelos de análisis de las tutorías destacan las actitudes hacia la misma y sus funciones tanto formativas como socializadoras. Pero, a nivel internacional, el abanico de modelos y enfoques es más amplio y va desde el grado de satisfacción con la tutoría de maestros y alumnos, estrategias de gestión e intervención desde la autoevaluación y heteroevaluación de su situación docente ante la tutoría, y en experiencias de implementación de las tutorías basadas en sus funciones académicas, más que en sus funciones afectivas; asimismo, desde un enfoque como herramienta de aprendizaje y mejora de rendimiento, más que en la revisión de un componente afectivo emocional. Sin embargo, el diseño de instrumentos de medición de la tutoría, tanto desde los enfoques nacionales e internacionales en educación superior, destacan la importancia de que estos reflejen las demandas y necesidades de los estudiantes y les permita a los tutores monitorear o mejorar la función tutorial.

\section{Metodología}

El método utilizado en el estudio fue de tipo cuantitativo con un diseño de estudio instrumental, que se caracteriza por el desarrollo de pruebas e instrumentos, incluyendo tanto el diseño como su adaptación (Montero, León, 2002). Se han considerado como pertenecientes a esta categoría todos los estudios encaminados al desarrollo de pruebas y aparatos, incluyendo tanto el diseño como su adaptación al software Statistical Package for Social Science (SPSS) versión 24, y el software Structural Equation Modeling (AMOS) versión 24.

\section{Muestra}

La muestra estuvo conformada por 343 estudiantes universitarios, 222 estudiantes de la Facultad de Trabajo Social de la Universidad Autónoma de Coahuila, del primero, segundo, tercero, cuarto, quinto, séptimo y octavo semestres de la licenciatura en Trabajo Social, y 121 estudiantes de la licenciatura de Trabajo Social de la Universidad Autónoma de Aguascalientes.

\section{Instrumentos}

Para la realización del estudio se conformó un instrumento de medida denominado Escala de Actitudes Hacia la Tutoría, utilizando tres escalas de medida que pasaron por un procedimiento 
de validez y confiabilidad de forma independiente, y posteriormente de manera conjunta, las cuales se describen a continuación:

1. Escala de Actitudes hacia la Tutoría (EAHT) de Caldera, Carranza, Jiménez y Pérez, (2015): se utilizó en su versión final de 16 ítems integrados en tres factores correlacionados. El Alpha de Cronbach de la prueba reportada de esta escala fue $a=0.895$. La confiablidad de la escala reportó una consistencia interna de $a=.871$ para el factor "Creencias sobre la Tutoría", de $a=.805$ para el de "Afectos sobre la Tutoría" y a = .683 para el de "Disposiciones ante la Tutoría".

2. Escala de actitudes de los estudiantes universitarios hacia las tutorías académicas, desarrollada por Solaguren-Beascoa y Moreno (2016) con 18 reactivos; la escala está conformada por tres dimensiones: aspectos de seguridad o confianza, de motivación o interés personal y de utilidad para la asignatura. Sin embargo, para este estudio se utilizó la medida unidimensional considerando que la escala formada por todos los ítems presenta un Alpha de Cronbach menor, aunque aceptable, de valor $a=0.63$, por lo que la escala global puede interpretarse como unidimensional (actitud general hacia las tutorías académicas).

3. Escala de percepción de la tutoría afectiva: Se elaboraron 15 reactivos estableciendo como constructo de medida a la tutoría afectiva. El abordaje de la tutoría afectiva utilizada en este estudio surge a partir de la revisión y análisis de diversos modelos, enfoques y prácticas de la tutoría, además de tomar en cuenta las experiencias propias y de otras universidades que implementan programas de tutorías, en donde se pudo observar que existen prácticas útiles y necesarias enfocadas a una tutoría más personalizada, la cual se define como tutoría afectiva. De esta manera, en la escala de tutoría afectiva se pueden identificar elementos como la felicidad que le genera al tutorado tener a su tutor, así como el clima de confianza que el tutorado siente con el tutor para abordar cuestiones personales, e incluso la influencia o motivación que los consejos del tutor tienen sobre el tutorado para tomar decisiones importantes de su vida. Algunos de los elementos que se encuentran dentro de los constructos de la escala esbozan los pensamientos de los tutorados con respecto a los tutores, describiéndolos como empáticos, con herramientas para ayudarlos a solucionar situaciones de conflicto, les brindan confianza, y le dan seguimiento a las situaciones personales y académicas que se están tratando en la tutoría.

Para la conformación de la escala total de actitudes hacia la tutoría se siguieron dos procesos: 1 . El análisis factorial exploratorio (AFE) de cada escala para la obtención de los reactivos válidos y del coeficiente de confiabilidad Alpha de Cronbach de cada escala por separado y, posteriormente, el AFE del total de reactivos que obtuvieron los valores aceptables en el primer paso, para conformar la escala total de actitudes hacia la tutoría; 2. El análisis factorial exploratorio desde el método de componentes principales basado en autovalor, para determinar el comportamiento de las dimensiones en el interior de la escala total de actitudes hacia la tutoría y el análisis factorial confirmatorio de la escala total de actitudes hacia la tutoría para confirmar 
las dimensiones de la misma, teniendo como base del modelo lo que indicó inicialmente el AFE. A partir de esos análisis finales, la escala total de actitudes hacia la tutoría reveló dos factores: 1. Confianza en la tutoría afectiva y 2. Utilidad en la tutoría académica.

\section{Procedimiento}

Esta investigación tuvo por escenario la Facultad de Trabajo Social de la Universidad Autónoma de Coahuila y el Departamento de Trabajo Social de la Universidad Autónoma de Aguascalientes. Se validaron tres escalas de medición por medio del método de extracción de componentes principales en un análisis factorial exploratorio y un análisis factorial confirmatorio por medio del SPSS versión 25 y el AMOS versión 20, teniendo adecuados coeficientes de confiabilidad, porcentajes de varianza explicada e índices de ajuste adecuados para determinar los instrumentos como válidos y aceptables (véase tabla 1); además, se obtuvieron cargas factoriales superiores a .4 como estándar establecido para la incorporación de los reactivos como válidos. La encuesta tuvo la característica de ser autoadministrada, y se aplicó en dos universidades públicas del norte y centro de México, específicamente a estudiantes de la licenciatura en Trabajo Social. Las encuestas fueron tomadas en las ciudades de Saltillo, Coahuila, y Aguascalientes, Aguascalientes, México. A los participantes se les solicitó su consentimiento para ser parte del estudio y se les informó sobre el uso estadístico y la divulgación de los resultados. El llenado de la encuesta le tomaba entre 20 y 30 minutos a las participantes. Esta investigación fue aprobada por la comisión de evaluación de la Facultad de Trabajo Social de la Universidad Autónoma de Coahuila.

\section{Análisis de los datos}

Se manejó el coeficiente Alpha de Cronbach para evaluar la consistencia interna de los reactivos del instrumento, donde valores inferiores a 0.69 son considerados insuficientes, en el rango de 0.70 a 0.79 son aceptables, entre 0.80 a 0.89 son buenos puntajes y aquellos superiores a 0.90 son considerado excelentes (Cronbach, 1951). La validez de constructo de la escala de tutorías académico-afectiva y de apoyo se evaluó con un modelo de ecuaciones estructurales utilizando el Análisis Factorial Confirmatorio (AFC). Se utilizaron los indices: Root Mean Square Error of Approximation (RMSEA) (Steiger, 1990); Comparative Fit Index (CFI) (Bentler, 1990); Normed Fit Index (NFI) (Bentler, Bonett, 1980) y Non-Normed Fit Index (NNFI) (Bentler, Bonett, 1980). En este sentido, se considera que el ajuste es adecuado cuando el RMSEA se sitúa en un valor igual o inferior a 0.08 (MacCallum et al., 2008; Marsh et al., 2004; Fan, Sivo, 2005), y cuando CFI, NFI, y NNFI sean iguales o superiores a 0.95 (Hu, Bentler, 1999). La validez convergente se obtuvo a través de la correlación bivariada entre los reactivos de la escala de Tutoría académica y tutoría afectiva, y para interpretar el tamaño del efecto se siguieron las recomendaciones de Cohen (1992), en donde $r<0.10$ se considera un efecto pequeño, $r=0.30$ es un tamaño del efecto mediano y $r=$ 0.50 es un tamaño del efecto grande. 


\section{Resultados}

Se establecieron las medidas de tendencia central de los ítems: media, desviación típica, moda y mediana. Kline (2011) determina que los valores con puntos de corte entre 3.0 y 10 para la asimetría y la curtosis aseguran la normalidad univariada de los datos. La asimetría y la curtosis estuvieron dentro del rangos aceptables. De la subescala de confianza en la tutoría afectiva, el reactivo que obtuvo la media más alta fue el número 62 "Me siento feliz con mi tutor", mientras que el que obtuvo la media de menor valor fue el reactivo número 55 "Le platico todos mis problemas a mi tutor". De la subescala sobre la utilidad de la Tutoría académica, el reactivo que obtuvo la media más alta fue el número 34 "La tutoría es una práctica educativa que debe perdurar", mientras que el que obtuvo la media de menor valor fue el reactivo número 42 "Las tutorías son estimulantes para afrontar los estudios" (véase tabla 1). La asimetría fue baja en la mayoría de las variables, así como la curtosis que fue tendiente a una forma mesocúrtica.

Tabla 1. Medidas de tendencia central de los ítems de la escala de Confianza en la Tutoría Afectiva y Utilidad en la Tutoría Académica

\begin{tabular}{|c|c|c|c|c|c|c|}
\hline Escala de Confianza en la Tutoría Afectiva & $\mathrm{M}$ & $\mathrm{DE}$ & Asimetría & Curtosis & Mín. & Máx. \\
\hline 57-El clima entre el tutor y yo es propicio para comunicarnos & 3.95 & 1.16 & -0.972 & 0.139 & 1 & 5 \\
\hline 62-Me siento feliz con mi tutor & 4.12 & 1.156 & -1.318 & 0.981 & 1 & 5 \\
\hline 50-Me ayuda a tomar decisiones & 3.44 & 1.123 & -0.312 & -0.402 & 1 & 5 \\
\hline 51-Me ayuda a solucionar problemas personales & 3.04 & 1.307 & -0.018 & -1.017 & 1 & 5 \\
\hline 53-Me ayuda a entender la dinámica de la escuela & 3.88 & 1.033 & -0.754 & 0.094 & 1 & 5 \\
\hline 49-Me siento con mucha confianza & 3.84 & 1.056 & -0.592 & -0.323 & 1 & 5 \\
\hline 52-Me ayuda a solucionar problemas académicos & 3.81 & 1.092 & -0.68 & -0.177 & 1 & 5 \\
\hline 59-El tutor es empático conmigo para comprender lo que me pasa & 3.85 & 1.185 & -0.896 & 0.034 & 1 & 5 \\
\hline 55-Le platico todos mis problemas a mi tutor & 2.51 & 1.319 & 0.529 & -0.83 & 1 & 5 \\
\hline 61-El tutor me canaliza con expertos para atender mis problemas & 3.03 & 1.488 & -0.035 & -1.363 & 1 & 5 \\
\hline 56-El tutor respeta mi horario para la tutoría & 3.99 & 1.215 & -1.04 & 0.096 & 1 & 5 \\
\hline 37-Me siento cómodo cuando acudo con mi tutor & 3.71 & 1.198 & -0.763 & -0.249 & 1 & 5 \\
\hline 45-Las tutorías son un medio eficaz para resolver dudas sobre mi vida & 3.23 & 1.216 & -0.244 & -0.797 & 1 & 5 \\
\hline 41-La tutoría me ayuda a entender algunos contenidos de asignaturas & 3.44 & 1.157 & -0.413 & -0.577 & 1 & 5 \\
\hline 54-El tutor muestra mucho interés en mis asuntos & 3.72 & 2.54 & 11.9 & 189.817 & 1 & 5 \\
\hline Escala de Utilidad en la Tutoría Académica & $\mathrm{M}$ & $\mathrm{DE}$ & Asimetría & Curtosis & Mín. & Máx. \\
\hline 27-La tutoría académica mejora la calidad de la facultad de trabajo social & 3.57 & 0.907 & -0.477 & 0.31 & 1 & 5 \\
\hline 29-Creo que la tutoría facilita la adaptación de los estudiantes al ambiente escolar & 3.56 & 0.944 & -0.439 & 0.021 & 1 & 5 \\
\hline 32-Me siento satisfecho con el programa de tutoría de la facultad de trabajo social & 3.55 & 1.037 & -0.499 & -0.134 & 1 & 5 \\
\hline 34-La tutoría es una práctica educativa que debe perdurar & 3.79 & 0.99 & -0.746 & 0.225 & 1 & 5 \\
\hline 42-Las tutorías son estimulantes para afrontar estudios & 3.44 & 1.012 & -0.273 & -0.279 & 1 & 5 \\
\hline 26-La tutoría académica contribuye en mi formación integral como trabajador (a) social & 3.64 & 0.953 & -0.537 & 0.286 & 1 & 5 \\
\hline
\end{tabular}

Nota: Elaboración propia.

\section{Consistencia interna de la escala}

En el análisis factorial exploratorio de la escala total de actitudes hacia la tutoría obtuvo un valor de medida Kaiser-Meyer Olkin de adecuación de muestreo $.925(\mathrm{KMO}=.925)$, que se considera como muy buena adecuación. La prueba de esfericidad de Bartlett rechazó la hipótesis nula de independencia entre las variables $\left(X^{2}=3499.810, g l=210 ; p \leq .000\right)$. Inicialmente se procesa la 
varianza explicada de la escala total de actitudes hacia la tutoría, que de acuerdo con el AFE fue de $40.30 \%$ y un coeficiente de confiabilidad de Alpha de Cronbach de .90 con 21 reactivos.

En el análisis factorial exploratorio, la reducción de dimensiones con el método de extracción de componentes principales arrojó una solución para dos factores rotados desde el método de varimax. El primer factor, denominado Confianza en la tutoría afectiva y el segundo factor denominado Utilidad en la tutoría académica, obtuvieron niveles de varianza total explicada para una solución de dos factores, de $44.69 \%$ para la escala de Confianza en la tutoría afectiva, con un coeficiente de confiabilidad Alpha de Cronbach de .88 , con 15 reactivos y, posteriormente, para la escala Utilidad en la tutoría académica, una varianza total explicada para una solución de dos factores, de 59.14\%, con un coeficiente de confiabilidad Alpha de Cronbach de .85, con 6 reactivos.

La escala de confianza en la tutoría afectiva obtuvo un valor de medida Kaiser-Meyer Olkin de adecuación de muestreo .921 , que se considera como muy buena adecuación. La prueba de esfericidad de Bartlett rechazó la hipótesis nula de independencia entre las variables $\left(X^{2}=2306.556\right.$, $\mathrm{gl}=105 ; \mathrm{p} \leq .000)$. En el análisis factorial exploratorio de la escala de utilidad de la tutoría académica se obtuvo un valor de medida Kaiser-Meyer Olkin de adecuación de muestreo .856, que se considera como muy buena adecuación. La prueba de esfericidad de Bartlett rechazó la hipótesis nula de independencia entre las variables $\left(x^{2}=887.824, g l=15 ; p \leq .000\right)$. 
Tabla 2. Matriz de componentes rotados y coeficientes de confiabilidad Alpha de Cronbach

\begin{tabular}{|c|c|c|}
\hline & Componentes & \multirow[t]{2}{*}{$\Omega$} \\
\hline & 12 & \\
\hline PER857 El clima entre el turor y yo es propicio para comminicantos & .778 & .870 \\
\hline PER1262 Me sivnto feliz con mi tutor & .753 & .872 \\
\hline PER350 Me ayoda a toenar decisiones: & .727 & .872 \\
\hline CEA9S1-Me ayoda a solucionar problemas personales & .725 & .871 \\
\hline PER453 Me ayvoda a entender la dinámica de la escuela & .723 & .873 \\
\hline CEAR49 Me sicneo coe mucha soeflanca & .722 & .874 \\
\hline CEA $1052 \mathrm{Me}$ ayuda a solucionar problemsas académicos & .711 & 872 \\
\hline PER959 El tutor es empitico conmigo pars comprender lo que me pasa & .671 & 876 \\
\hline PER6s5 Le platico todoc mis problemas a mi tuter & .591 & .879 \\
\hline PER I I6] EI tutor me canaliza con expertos para atender mis problemss & .508 & 885 \\
\hline PER756 El tutor respeta mi horario para la tutoria & .501 & 879 \\
\hline AHTI 137 Me siento cónsodo cuando acudo con mi tuitor & .457 & $.8 \times 2$ \\
\hline CEA545 Las tusorias son un medio eficaz para resolver dudas sobre mi vida & 452 & .879 \\
\hline CEA241La futoria me ayuda a entender algunos contenidos de asignaturas & 452 & 878 \\
\hline PER554 E1 tutor muestra mucho imerés en mis asuntod & 440 & .904 \\
\hline AHT227 La tutoria académica mejors la calicad de lo facultad de trabojo social & 819 & .823 \\
\hline AHT329 Creo que la futeria faxilita la adaptación de los estuxtiantes al ambiente escolar & 806 & .825 \\
\hline AHT126 La tatoria académica contribuye $\mathrm{cm}$ mi formacién integral como trabajados $(a)$ social & 795 & .822 \\
\hline AHT632 Me siento satisfecho con el programa de tutoria de la faculad de trabajo social & .719 & .824 \\
\hline AHT834 La tatoría es una práctica edacativa que debe perdarar & 707 & .833 \\
\hline CEA342 Las tuborias son estimulantes gare afrontar estudios & 465 & .867 \\
\hline
\end{tabular}

\section{Validez de constructo: Análisis Factorial Exploratorio y confirmatorio}

Se verificó la existencia de datos ausentes o débiles y se evidenciaron los casos de datos atípicos. Inicialmente, la escala general de la utilidad de la tutoría estaba conformada por 36 reactivos; sin embargo, en la primera revisión de su estructura interna y como parte del análisis factorial exploratorio de la escala completa, sólo se conservaron 25 reactivos en el primer ejercicio de extracción de factores, considerando el requisito de aceptación que establecen algunos autores sobre una estructura interna válida de reactivos con cargas factoriales mayores a .40 como estándar más riguroso, en comparación con cargas menores y la evaluación de la distribución normal univariante, a través de los estadísticos de asimetría y curtosis (Nunnally, 1970; Bandalos, Finney, 2010; Gaskin, Happell, 2014; Lloret-Segura, Ferreres-Traver, Hernández-Baeza, Tomás-Marco, 2014; Carmines, Zeller, 1979). Para identificar la pertinencia de los datos de la muestra se utilizó el índice de medida de adecuación de la muestra KMO y el test de esfericidad de Barlett (De la Fuente, 2011).

Posteriormente, en un segundo ejercicio de extracción de factores fueron eliminados dos reactivos más, siguiendo estos mismos criterios. La medida Kaiser-Meyer-Olkin de adecuación de muestreo fue de .925 , con un porcentaje de varianza explicada de 40.30 al eliminar los reactivos con cargas menores a .40. Además, esta escala general tuvo buena confiabilidad, con un coeficiente Alpha de Cronbach de .907 con 21 reactivos y siguiendo los criterios de algunos autores (Nunnally, 1970). Utilizando el método de rotación varimax y el método de componentes 
principales como método de extracción, esta escala general fue sometida a un tercer ejercicio de análisis factorial exploratorio. Se identificaron tres factores, sin embargo, fue necesario eliminar uno de ellos por la baja confiabilidad en el coeficiente Alpha de Cronbach $(<.7)$, posterior a la rotación de factores, esta escala de tres factores obtuvo una medida Káiser Meyer Olkin de adecuación de muestreo de .919 y con una varianza explicada de 48.40, con un coeficiente Alpha de Cronbach de la escala general de .902 con 18 reactivos de los 20 reactivos iniciales. Finalmente, la escala general quedó representada en dos factores: Factor 1. Confianza en la Tutoría Afectiva, con $44.67 \%$ varianza explicada y formada por 15 reactivos con un coeficiente de confiabilidad Alpha de Cronbach de .88. Factor 2. Utilidad en la Tutoría Académica, con 59.14\% de varianza explicada, extracción de cargas al cuadrado, un coeficiente de confiabilidad Alpha de Cronbach de .85 con 6 reactivos (véase tabla 2).

\section{Análisis Factorial Confirmatorio}

El estudio de la estructura interna también se llevó a cabo mediante un análisis factorial confirmatorio. Primero se calcularon estadísticos descriptivos para los reactivos de la escala: media, desviación típica, asimetría y curtosis. El análisis de la asimetría y curtosis, junto con el coeficiente de normalidad multivariada, ayudaron en la decisión del método más adecuado de estimación más apropiado. Se utilizó el método de máxima verosimilitud, también se usaron índices de ajuste del modelo y puntos de corte recomendados en la literatura (Hu, Bentler, 1999), estadístico chi-cuadrado $\left(X^{2}\right)$, índice de ajuste comparativo (CFI), raíz del residuo estandarizado medio (SRMR), error cuadrático medio de aproximación (RMSEA) y sus intervalos de confianza al $90 \%$ (IC 90\%). Considerándolos en conjunto, valores de CFI $\geq .95$, así como RMSEA y SRMR $\leq .08$ brindarían evidencia de un adecuado ajuste del modelo.

Para obtener evidencias internas de validez convergente se realizó un análisis de varianza promedio extraída del factor. Se calculó el tamaño del efecto de las correlaciones a partir del valor del coeficiente de correlación $>.20$ mínimo recomendado, $>.50$ moderado $>.80$ fuerte, y sus respectivos intervalos de confianza (Caycho-Rodríguez, 2017). Al llevar a cabo el modelamiento de ecuaciones estructurales, los investigadores a menudo primero evalúan el modelo de medida (si las variables reflejan con precisión las construcciones deseadas o factores) antes de evaluar el modelo estructural (Brown, 2006). En este sentido, se utilizó el enfoque en dos pasos propuesto por Gerbing y Anderson (1988): primero, se calculó un modelo de medición para dar validez de constructo a las diferentes dimensiones de la Escala General de la Utilidad y Efectividad de la Tutoría.

En segundo lugar, se formuló un modelo de regresión estructural que analizó la relación entre los diferentes constructos; ante este procedimiento siete reactivos fueron eliminados para establecer un mejor ajuste del modelo, por lo que de los 21 reactivos fue posible ajustar 14 . Se consideró una serie de coeficientes de ajuste para evaluar la bondad de ajuste de los modelos 
de medida con los datos empíricos. Por lo tanto, con base en los estudios de diferentes autores (Bentler, 1990; Bollen, Long, 1993; McDonald, Marsh, 1990), los índices de ajuste que se consideraron para evaluar el modelo estructural fueron: 2, 2 / df, RMSEA (Root Mean Square Error of Approximation), RMSR (Root Mean Square Residual) y los índices incrementales (CFI, IFI y TLI). En estos índices de bondad de ajuste se consideran aceptables los índices incrementales cuando son mayores que .90 y los índices de error (RMSEA y RMSR) son menores que .08 (Hu, Bentler, 1999; Byrne, 2012). Si la bondad de ajuste es adecuada, el modelo aboga por la plausibilidad de las relaciones postuladas entre las variables; si es inadecuada, la plausibilidad de tales relaciones es rechazada.

Las escalas obtienen una Chi cuadrada de 209.089, con 76 grados de libertad, $p=0.000, z=$ 597. El modelo resultó significativo $(P<.05)$, la discrepancia mínima resultó un índice razonable (CMIN/DF = 2.751) considerando las indicaciones de Marsh y Hocevar (1985). El índice de ajuste comparativo identificado por Bentler (1990) y que es idéntico al determinado por McDonald y Marsh (1990), obtuvo un puntaje alto y adecuado $(C F I=.932)$. El coeficiente de Tucker Lewis (Bollen, 1989) y el índice de ajuste incremental fueron altos (TLI = .906; IFI = .933) (Bollen, 1989). El índice de ajuste normado determinado por Bentles y Bonet en 1980 también fue adecuado (NFI= .900). Por otro lado, la aproximación de error de la raíz cuadrada (RMSEA), determinado por Browne y Cudeck (1993) como favorable con valores menores a .08, podría indicar un ajuste cercano del modelo en relación con los grados de libertad. En este modelado se obtuvo un error de la raíz cuadrada adecuada (RMSEA $=.072$ ). Todas las relaciones entre reactivos resultaron significativas (véase la figura 1). 
Figura 1. Modelo de ecuaciones estructurales de dos constructos de medida de la confianza y utilidad de la tutoría. El modelo está constituido por la de Confianza en la Tutoría Afectiva (CTA), y Utilidad en la tutoría académica (UTA)

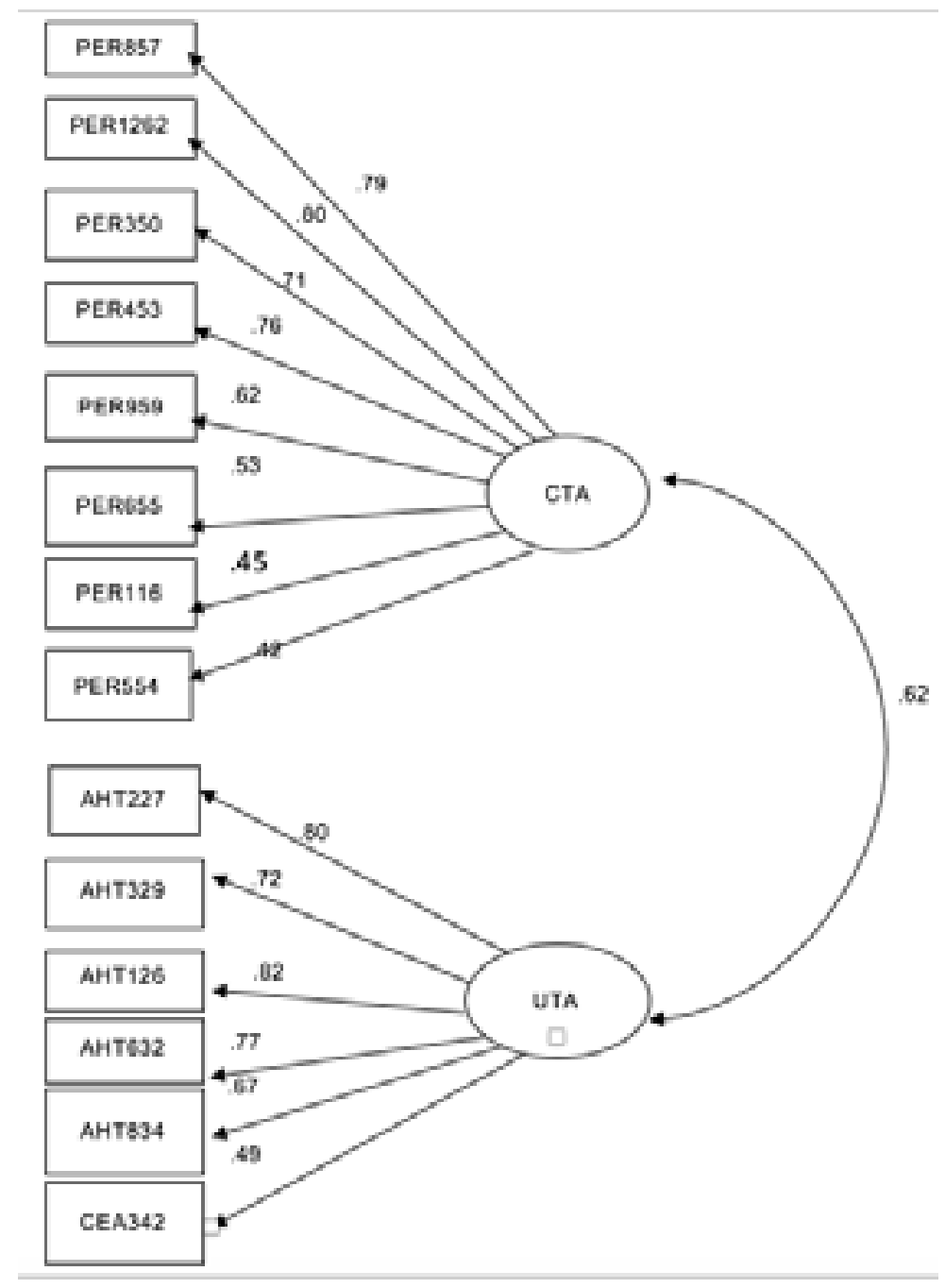

\section{Validez convergente}

La correlación entre las puntuaciones de la escala de Tutoría académico-afectiva. Todas las relaciones entre los diferentes reactivos de la escala total mantuvieron niveles significativos de correlación, por lo que se puede confirmar la asociación interna de los reactivos (véase la tabla 3). 
Tabla 3. Correlaciones Bivariadas entre los reactivos de la escala de Tutorías Académicas y Tutorías afectiva

\begin{tabular}{|c|c|c|c|c|c|c|c|c|c|c|c|c|c|c|c|c|c|c|c|c|}
\hline & 57 & 62 & 50 & 51 & 53 & 49 & 52 & 59 & 55 & 61 & 56 & 37 & 45 & 41 & 54 & 27 & 29 & 26 & 32 & 34 \\
\hline 62 & $.679^{* *}$ & 1 & & & & & & & & & & & & & & & & & & \\
\hline 50 & $.532^{* *}$ & $.546^{* *}$ & 1 & & & & & & & & & & & & & & & & & \\
\hline 51 & $.514^{* *}$ & $.488^{* *}$ & $.698 * *$ & 1 & & & & & & & & & & & & & & & & \\
\hline 53 & $.547 * *$ & $.604 * *$ & $.600 * *$ & $.575^{* *}$ & 1 & & & & & & & & & & & & & & & \\
\hline 49 & $.579 * *$ & $.617^{* *}$ & $.614^{* *}$ & $.519^{* *}$ & $.524 * *$ & 1 & & & & & & & & & & & & & & \\
\hline 52 & $.540^{* *}$ & $.567^{* *}$ & $.705 * *$ & $.615^{* * *}$ & $.755^{* *}$ & $.522 * *$ & 1 & & & & & & & & & & & & & \\
\hline 59 & $.527^{* *}$ & $.525^{* *}$ & $.396 * *$ & $.417^{* * *}$ & $.445^{* *}$ & $.478^{* *}$ & $.390^{* *}$ & 1 & & & & & & & & & & & & \\
\hline 55 & $.433^{* *}$ & $.376^{* *}$ & $.436^{* *}$ & $.601^{* *}$ & $.406^{* *}$ & $.445^{* *}$ & $.409 * *$ & $.294 * *$ & 1 & & & & & & & & & & & \\
\hline 61 & $.364 * *$ & $.325^{* *}$ & $.313^{* *}$ & $.373^{* *}$ & $.360^{* *}$ & $.283^{* *}$ & $.302 * *$ & $.298 * *$ & $.269^{* *}$ & 1 & & & & & & & & & & \\
\hline 56 & $.484 * *$ & $.387^{* * *}$ & $.303^{* *}$ & $.318^{* *}$ & $.447^{* *}$ & $.266^{* *}$ & $.449 * *$ & $.380^{* *}$ & $.173^{* * *}$ & $.174 * *$ & 1 & & & & & & & & & \\
\hline 37 & $.428^{* *}$ & $.509^{* *}$ & $.415^{* *}$ & $.299 * *$ & $.419 * *$ & $.454^{* *}$ & $.393 * *$ & $.330^{* *}$ & $.261^{* *}$ & $.154 * *$ & $.301 * *$ & 1 & & & & & & & & \\
\hline 45 & $.365^{* *}$ & $.319^{* *}$ & $.427 * *$ & $.406^{* *}$ & $.389^{* *}$ & $.326^{* *}$ & $.446^{* *}$ & $.286^{* *}$ & $.285^{* *}$ & $.227 * *$ & $.264 * *$ & $.345^{* *}$ & 1 & & & & & & & \\
\hline 41 & $.405^{* *}$ & $.377 * *$ & $.413^{* *}$ & $.377 * *$ & $.442 * *$ & $.312^{* *}$ & $.429^{* *}$ & $.255^{* *}$ & $.255^{* *}$ & $.236^{* *}$ & $.256^{* *}$ & $.353^{* *}$ & $.422 * *$ & 1 & & & & & & \\
\hline 54 & $.309^{* *}$ & $.319^{* *}$ & $.251^{* *}$ & $.260^{* *}$ & $.350^{* *}$ & $.292 * *$ & $.328 * *$ & $.310^{* *}$ & $.238^{* *}$ & $.250^{* *}$ & $.219^{* * *}$ & $.207 * *$ & $.272^{* *}$ & $.199 * *$ & 1 & & & & & \\
\hline 27 & $.309^{* *}$ & $.314^{* *}$ & $.308^{* *}$ & $.300^{* *}$ & $.377^{* *}$ & $.317^{* *}$ & $.369^{* *}$ & $.228^{* *}$ & $.187^{* * *}$ & $.190^{* *}$ & $.283^{* *}$ & $.339 * *$ & $.277^{* *}$ & $.274 * *$ & $.134 *$ & 1 & & & & \\
\hline 29 & $.245^{* *}$ & $.264 * *$ & $.290 * *$ & $.298 * *$ & $.303^{* *}$ & $.232 * *$ & $.311^{* *}$ & $.126^{*}$ & $.152^{* *}$ & $.122^{*}$ & $.124 *$ & $.277 * *$ & $.310^{* *}$ & $.301 * *$ & $.197 * *$ & $.592^{* *}$ & 1 & & & \\
\hline 26 & $.352^{* *}$ & $.378^{* *}$ & $.386^{* *}$ & $.371 * *$ & $.382^{* *}$ & $.380^{* *}$ & $.393 * *$ & $.260^{* *}$ & $.243 * *$ & $.172 * *$ & $.234^{* *}$ & $.384 * *$ & $.359^{* *}$ & $.292 * *$ & $.152^{* * *}$ & $.734^{* *}$ & $.570^{* *}$ & 1 & & \\
\hline 32 & $.472 * *$ & $.503^{* *}$ & $.408^{* *}$ & $.383^{* *}$ & $.473^{* *}$ & $.406^{* *}$ & $.480^{* *}$ & $.356^{* *}$ & $.345^{* *}$ & $.193^{* *}$ & $.335^{* *}$ & $.516^{* *}$ & $.384^{* *}$ & $.380^{* *}$ & $.224^{* *}$ & $.570^{* *}$ & $.519^{* *}$ & $.625^{* * *}$ & 1 & \\
\hline 34 & $.334 * *$ & $.290^{* *}$ & $.314^{* *}$ & $.279 * *$ & $.394 * *$ & $.298^{* *}$ & $.369^{* *}$ & $.184 * *$ & $.213^{* * *}$ & $.205^{* * *}$ & $.201 * *$ & $.381 * *$ & $.315^{* *}$ & $.329 * *$ & $.198 * *$ & $.513^{* *}$ & $.555^{* *}$ & $.476^{* *}$ & $.541^{* *}$ & 1 \\
\hline 42 & $.394 * *$ & $.346^{* *}$ & $.406^{* *}$ & $.356^{* *}$ & $.410^{* *}$ & $.368 * *$ & $.387^{* *}$ & $.223^{* *}$ & $.249 * *$ & $.206^{* *}$ & $.222 * *$ & $.307 * *$ & $.419^{* *}$ & $.566 * *$ & $.240^{* *}$ & $.300^{* *}$ & $.419^{* *}$ & $.338^{* *}$ & $.395^{* *}$ & $.415^{* *}$ \\
\hline
\end{tabular}

\section{Discusión}

Con respecto a la validación de la presente escala se puede señalar que, según los resultados previamente señalados, es una escala consistente, válida y confiable, en la medición de la "Confianza en la Tutoría Afectiva" y de la "Utilidad de la Tutoría Académica", factores que pueden ayudar a comprender cómo es la relación entre tutor y tutorado en las tutorías personales, y cómo también se percibe el programa de tutoría institucional, considerando elementos metodológicos que coinciden con las validaciones de escalas similares como las de León y Fernández (2019), De la Cruz y Abreu (2017) y López y Pantoja (2016) .

En este sentido, el factor de "Confianza en la Tutoría Afectiva" de la presente escala rescata la medición de elementos que den cuenta, como lo señala Canales (2010), de la relación "cara a cara" entre el docente tutor y el alumno tutorado, lo cual implica justamente abordar algunos elementos clave de la vida del tutorado que no sólo se circunscriben al ámbito académico, aunque ciertamente influyen en su desempeño escolar, coincidiendo con Beascoa y Moreno (2016), al hablar de entender la actitud de los estudiantes a partir de su seguridad y confianza, 
motivación e interés personal, además de la utilidad para la asignatura, al igual que con Ahumada y Obregón (2015), quienes posicionan la autoestima como un elemento esencial para el desempeño académico exitoso.

Este factor, además, brinda elementos para medir también la percepción que se tiene de parte de los estudiantes con respecto a la disposición y habilidad del tutor para realizar la tutoría, siendo este un aspecto importante que señalan Rubio y Martínez (2012), dado que el tutor es quien promueve o no un contexto de mutuo beneficio, que remite ineludiblemente a la creación de un lazo interpersonal o es solamente un requisito más con el que hay que cumplir, tal y como lo señalan Ayala, Naranjo y Castro (2014).

Por otra parte, el factor "Utilidad de la Tutoría Académica" proporciona elementos que posibilitan medir la percepción que tienen los estudiantes con respecto a su Programa Institucional de Tutoría, ofreciendo elementos para evaluar el impacto que tiene en su vida académica y formación profesional, coincidiendo con los esfuerzos de evaluación de programas de tutorías realizados por Sánchez, Amador y Castillo (2011), y Ayala, Naranjo y Castro (2014). Lo anterior, justamente, para contrastar si realmente las tutorías son un espacio que habilita al tutorado a tomar resoluciones que "le aseguren el éxito escolar y profesional" tal y como señala Canales (2010), y previniendo la reprobación y la deserción, según Hernández, Martínez y Carranza (2013), y coincidiendo también con Guerra, Lima y Lima (2017) al señalar que las tutorías deben "fomentar buenos hábitos de estudio", mediante una atención personalizada que se ajuste a sus necesidades, de acuerdo con Andreucci y Curiche (2017), y que además convierta a quienes estudian en la universidad en individuos competentes que puedan desenvolverse adecuadamente en "ambientes dinámicos y complejos", como señalan Sánchez, Amador y Castillo (2011).

Todo lo anterior es sustentado en la perspectiva del estudiante, a quienes justamente se busca beneficiar con la implementación de estos programas de acompañamiento, proporcionándonos así elementos de evaluación desde el impacto que percibe el usuario final, coincidiendo en este sentido con el enfoque desde la percepción de los tutorados de la investigación realizada por Zaldívar, Nava y Lizárraga (2018).

Finalmente, cabe señalar que dicha escala puede seguirse trabajando en futuras investigaciones al ampliar la muestra hacia otras escuelas, facultades e instituciones, tanto de Trabajo Social como de otras carreras profesionales, dado que puede haber diferencias importantes entre ellas, como lo ha señalado Gómez-Collado (2012).

\section{Conclusiones}

Este estudio propone una escala para medir la efectividad de la tutoría universitaria en términos de afectividad, en donde el tutor acompaña al tutorado en el tránsito académico de su carrera profesional, brindándole apoyo académico, administrativo, pero además siendo afectivo a través de la empatía con las situaciones personales que sus tutorados tienen, para generar un 
clima de confianza al momento de la tutoría en donde el tutorado lo percibe y comparte sus situaciones con su tutor porque siente que éste lo apoyará con herramientas que le permitirán afrontar cualquier obstáculo a lo largo de su profesión.

En este sentido, se propone una escala que mide justamente la orientación de las tutorías más personalizadas que ejercen los docentes tutores, porque si bien algunos institutos, facultades o escuelas que implementan programas de tutorías, debido al número de tutorados que se les asignan, puede quedar inviable una tutoría personalizada desde la afectividad y se opta por hacerlas generalmente de manera grupal. En lo operativo, la tutoría representa un reto en la educación, en donde el tutor cuenta con horario establecido para las tutorías y en ocasiones este horario se ver rebasado por las exigencias de la operatividad del programa o de las tutorías; en ocasiones, esto obstaculiza el funcionamiento de los programas institucionales de tutorías. Para garantizar la efectividad de las tutorías se recomienda que, en medida de lo posible, los tutores diagnostiquen los perfiles de cada uno de sus tutorados a fin de implementar tutorías de acuerdo a las necesidades de cada uno, de este modo la tutoría afectiva será para aquellos alumnos que presenten más necesidad de acompañamiento de un tutor, mientras que los tutorados más autónomos tendrán a un tutor que supervise su tránsito académico y que cuando sienta la necesidad de abordar alguna situación personal, sabe que el tutor lo apoyará.

Dado que el ejercicio de la tutoría académica implica la interacción con emociones y situaciones conflicto de los tutorados, se recomienda que las escuelas realicen programas de seguimiento y capacitación en sus tutores, a fin de evitar la presencia de problemas relacionados con la salud mental y equilibrio emocional. Desde la investigación se sugiere trabajar una escala dirigida a los tutores para analizar cómo perciben al programa de las tutorías y, en general, saber qué tipo de tutoría es la que más implementan.

\section{Referencias}

Ahumada, G.; N. Obregón (2015). La tutoría profesional: una estrategia para favorecer la formación de docentes a partir del desarrollo de la autoestima. Ra Ximhai, 11(4), 75-90. 10.35197/rx.11.01.e2.2015.04.fa

Andreucci, P.; A. Curiche (2017). Tutorías académicas: Desafíos de un programa piloto entre pares en una universidad no selectiva. Revista Latinoamericana de Ciencias Sociales Niñez y Juventud, 15(1), 357-371. 10.37954/se.v5i1.56

Ayala, B.; C. Naranjo; B. Castro (2014) Evaluación del programa de tutorías del Instituto Tecnológico de Los Mochis (caso carrera de ingeniería en gestión empresarial período 2013-2014). Ra Ximhai, 10(5), 187-198. 10.35197/rx.10.03.e1.2014.13.na 
Bandalos, D.; S. Finney (2010). Factor Analysis: Exploratory and Confirmatory. En Hancock; G. R. Mueller (eds.). Reviewer's Guide to Quantitative Methods. Routledge: Nueva York, 93-114. 10.4324/9780203861554

Bentler, K. (1990) Einleitung. En Bentler, K. (ed.). Grundlagen der Investitionsentscheidung über automatische Formanlagen. fir+iaw Forschung für die Praxis. Berlín, Heidelberg: Springer, 1-7. 10.1007/978-3-642-95641-6

Bentler, P.; D. Bonett (1980). Significance Tests and Goodness of Fit in the Analysis of Covariance Structures. Psychological Bulletin, 88(3), 588-606. 10.1037/0033-2909.88.3.588

Brown, T. A. (2006). Confirmatory Factor Analysis for Applied Research. Nueva York: Guilford Press. Browne, M.; R. Cudeck (1993). Alternative Ways of Assessing Model Fit. En Bollen, K.; J. Long (eds.). Testing Structural Equation Models. Newbury Park: Sage, 136-162.

Bricall, J. (2000). Informe Universidad 2000. Barcelona: CRUE.

Bollen, K. (1989). Structural Equations with Latent Variables. Nueva York: John Wiley \& Sons.

Bollen, K.; J. Long (1993). Testing Structural Equation Models. Newbury Park: Sage.

Caldera, J.; A. Carranza; A. Jiménez Padilla; I. Pérez Pulido (2015). Actitudes de los estudiantes universitarios ante la tutoría. Diseño de una escala de medición. Revista de la Educación Superior, XLIV(1), (173), 103-124. http://www.redalyc.org/articulo.oa?id=60436859005

Canales, E. (2010). El perfil del tutor académico. México: Universidad Autónoma del Estado de Hidalgo. https://www.uaeh.edu.mx/investigacion/productos/4614/

Carmines, E.; R. Zeller (1979). Reliability and Validity Assesment. Beverly Hills: Sage Publications.

Caycho-Rodríguez, T. (2017). Effect Size and Confidence Intervals for Correlations: Contributions to Montes Hidalgo y Tomás-Sábado. Enfermería Clínica (English Edition), 27(5), 331-332. 10.1016/j.enfcli.2017.07.001

Chiva, S.; S. Ramos (2015). Diseño y validación de una escala para evaluar las estrategias de gestión e intervención docente en educación primaria. Actualidades Investigativas en Educación.

Cohen, J. (1992). Statistical Power Analysis. SAGE Journals. 1(3), 98-101. 10.1111/1467-8721. ep10768783

Cortada, N. (1999). Teorías psicométricas y construcción de tests. Buenos Aires.

Cronbach, L. (1951). Review of The Bender-Gestalt Test: Quantification and Validity for Adults [Pascal, G.; B. Suttell. Review of the book the Bender-Gestalt test: Quantification and validity for adults]. Journal of Educational Psychology, 42(7), 438-439. https://doi.org/10.1037/ $\underline{\mathrm{h} 0053236}$

De la Cruz, F.; L. Abreu (2017). Evaluación de la tutoría en los estudios de posgrado: construcción y validez de escalas. REDU. Revista de Docencia Universitaria, 15(1), 11-36. 10.4995/ redu.2017.5682 
Fernández, S. (2011). Análisis factorial. Madrid: Universidad Autónoma de Madrid. http://www. fuenterrebollo.com/Economicas/ECONOMETRIA/MULTIVARIANTE/FACTORIAL/analisis-factorial.pdf

Fan, X.; A. Sivo (2005). Sensitivity of Fit Indexes to Misspecified Structural or Measurement Model Components: Rationale of Two-Index Strategy Revisited. Structural Equation Modeling: A Multidisciplinary Journal, 12(3), 343-367. 10.1207/s15328007sem1203 1

Gaskin, C.; B. Happell (2014). On Exploratory Factor Analysis: A Review of Recent Evidence, an Assessment of Current Practice, and Recommendations for Future Use. International Journal of Nursing Studies, (51), 511-521. 10.1016/j.jinurstu.2013.10.005

Gerbing, D.; J. Anderson (1988). An Updated Paradigm for Scale Development Incorporating Unidimensionality and Its Assessment. Journal of Marketing Research 25(2), 186-192. 10.1177/002224378802500207

Gómez, M. (2012). La percepción de los estudiantes sobre el Programa de Tutoría Académica. Convergencia, 19(58), 209-233. http://www.scielo.org.mx/scielo.php?pid=S1405-14352012 000100009\&script $=$ sci abstract

Gómez, D.; B. Amador; J. Castillo (2011). Análisis del programa de tutorías implementado para los estudiantes de la licenciatura en mercadotecnia. CienciaUAT, 5(4), 54-59. https://www. redalyc.org/pdf/4419/441942924007.pdf

González A.; I. Avelino (2016). Tutoría: una revisión conceptual. Revista de Educación y DesarroIlo, (38), 57-68. http://www.cucs.udg.mx/revistas/edu desarrollo/anteriores/38/38 Gonzalez Palacios.pdf

Guerra, M.; M. Lima; J. Lima (2017). Effectiveness of Tutoring to Improve Academic Performance in Nursing Students at the University of Seville. Journal of New Approaches in Educational Research, 6(2), 93-103. 10.7821/naer.2017.7.201

Hernández, Y.; J. Martínez; C. Carranza (2013). Programa Institucional de Tutorías. Una estrategia para elevar el nivel de aprovechamiento en una institución educativa. Acta Universitaria, 1(23), 31-36. 10.15174/au.2013.395

Hu, L.; P. Bentler (1999). Cutoff Criteria for Fit Indexes in Covariance Structure Analysis: Conventional Criteria versus New Alternatives. Structural Equation Modeling: A Multidisciplinary Journal, 6(1), 1-55. 10.1080/10705519909540118

Kline, R. (2011). Principles and Practice of Structural Equation Modeling. Nueva York: Guilford Press. 10.1080/10705511.2012.687667

León, C.; D. Fernández (2019) Diseño y validación de una escala para evaluar el funcionamiento de las tutorías en Educación Secundaria. Revista de Investigación Educativa, 37(2), 525-541. 10.6018/rie.37.2.345251

Lloret-Segura, S.; A. Ferreres-Traver; A. Hernández-Baeza; I. Tomás-Marco (2014). El análisis factorial exploratorio de los ítems: una guía práctica, revisada y actualizada. Anales de Psicología, 30(3), 1151-1169. 10.6018/analesps.30.3.199361 
Lobato, C.; L. del Castillo; F. Arbizu (2005). Representations of University Tutoring in Teachers and Students: A Case Study. International Journal of Psycology and Psycological Therapy, 5(2), 148-168.

López C.; V. Pantoja (2016). Diseño y validación de una escala para comprobar la percepción y satisfacción de las familias andaluzas en relación con los procesos tutoriales en centros de educación primaria. Revista Española de Orientación y Psicopedagogía, 27(1). 10.5944/reop. vol.27.num.1.2016

MacCallum, J.; W. Bennett; D. Tieleman (2008). Distribution of Amino Acids in a Lipid Bilayer from Computer Simulations. Biophysical Journal, (94), 3393-3404. 10.1529/biophysj.107.112805

Martínez-Arias, R. (2005). Psicometría: teoría de los test psicológicos y educativos. Madrid: Editorial Síntesis.

Marsh, H.; Z. Wen; K. Hau (2004). Structural Equation Models of Latent Interactions: Evaluation of Alternative Estimation Strategies and Indicator Construction. Psychological Methods, 9(3), 275-300. 10.1037/1082-989x.9.3.275

McDonald, R.; H. Marsh (1990). Choosing a Multivariate Model: Noncentrality and Goodness of Fit. Psychological Bulletin, 107(2), 247-255. https://doi.org/10.1037/0033-2909.107.2.247

Montero, I.; O. León (2002). Clasificación y descripción de las metodologías de investigación en psicología. Revista Internacional de Psicología Clínica y de la Salud, 2(3), 503-508.

Nunnally, J. (1970). Introduction to Psychological Measurement. Japón: McGraw Hill.

Pérez, J.; P. Martínez; M. Martínez (2015). Satisfacción del estudiante universitario con la tutoría. Diseño y validación de un instrumento de medida. Estudios sobre Educación, (29), 81-101. https://revistas.unav.edu/index.php/estudios-sobre-educacion/article/view/3452

Ponce; S.; B. García; D. Islas; Y. Martínez; A. Serna (2018). De la tutoría a la mentoría. Reflexiones en torno a la diversidad del trabajo docente. Revista Páginas de Educación, 11(2), 215-235. 10.22235/pe.v11i2.1635

Romero, F.; R. Chávez; K. Sandoval (2014). Las tutorías como estrategia de fortalecimiento en el nivel superior. Ra Ximhai, 10(3), 75-86. https://www.redalyc.org/pdf/461/46131111006.pdf

Rubio, P.; J. Martínez (2012). La acción tutorial desde la perspectiva de los alumnos de la Universidad Autónoma de San Luis Potosí. Perfiles Educativos, 34(138), 28-45. http://www.scielo. org.mx/scielo.php?script=sci arttext\&pid=S0185-26982012000400003

Rumbo, B.; T. Gómez (2011). La acción tutorial en un contexto universitario masificado y la reivindicación europea de su valor formativo. Revista de Formación e Innovación Educativa Universitaria, 4(1), 13-34

Solaguren-Beascoa, M.; L. Moreno (2016). Escala de actitudes de los estudiantes universitarios hacia las tutorías académicas. Educación XXI, 19(1), 247-266. 10.5944/educXX1.14479

Solaguren-Beascoa, M.; L. Moreno (2019). Las tutorías académicas en carreras de ingeniería: una visión actual. Revista de Investigación Educativa, 37(1), 251-267. 10.6018/rie.37.1.320931 
Steiger, J. (1990). Structural Model Evaluation and Modification: An Interval Estimation Approach. Multivariate Behavioral Research, 25(2), 173-180. 10.1207/s15327906mbr2502 4

Tejedor, F.; A. García-Valcárcel (2007). Causas del bajo rendimiento del estudiante (en opinión de los profesores y alumnos). Propuestas de mejora en el EEES. Revista de Educación, (342), 443-473.

Vieira, M.; J. Vidal (2006). Tendencias de la educación superior europea e implicaciones para la orientación universitaria. Revista de Orientación y Psicopedagogía, 17(1), 75-97.

Zaldívar, A.; L. Nava; J. Lizárraga (2018). Influence of Tutoring on Mathematics Learning. Student Perspective. RIDE Revista Iberoamericana para la Investigación y el Desarrollo Educativo, 8(16). $\underline{10.23913 / \text { ride.v8i16.355 }}$ 